Pacific

Journal of

Mathematics

EQUIVARIANT EMBEDDINGS OF STEIN DOMAINS SITTING INSIDE OF COMPLEX SEMIGROUPS

BERNHARD KRÖTZ 


\title{
EQUIVARIANT EMBEDDINGS OF STEIN DOMAINS SITTING INSIDE OF COMPLEX SEMIGROUPS
}

\author{
BERNHARD KRÖTZ
}

\begin{abstract}
In this paper we prove an equivariant version of Hörmanders embedding theorem for Stein manifolds. More concretely, let $G$ be a connected Lie group sitting in its complexification $G_{\mathbb{C}}$ and $D \subseteq G_{\mathbb{C}}$ a $G \times G$-invariant Stein domain. Under slight obstructions on $D$ we construct a Hilbert space $\mathcal{H}$ equipped with a unitary $G \times G$-action and a holomorphic equivariant closed embedding $e: D \rightarrow \mathcal{H}^{*} \backslash\{0\}$.
\end{abstract}

\section{Introduction.}

An interesting problem in the field of equivariant complex analysis is: Given a connected Lie group $G$ sitting in its universal complexification $G_{\mathbb{C}}$, how do the $G \times G$-invariant Stein domains in $G_{\mathbb{C}}$ look like. K.-H. Neeb has shown in $[\mathrm{Ne} 98]$ that all domains of the form

$$
D=G \exp _{G_{\mathbb{C}}}\left(i D_{h}\right),
$$

where $D_{h} \subseteq \mathfrak{g}$ is a $\operatorname{Ad}(G)$-invariant convex domain consisting of elliptic elements, i.e., all operators $i$ ad $X, X \in D_{h}$, are diagonalizble over the reals, are Stein manifolds. Moreover there is also strong evidence for that these $D$ exhaust up to multiplication with $N_{G_{\mathbb{C}}}(G)$ all proper bi-invariant Stein domains in $G_{\mathbb{C}}$ (cf. [GG77], [Ne98]).

By Hörmander's Embedding Theorem one knows that every Stein manifold of dimension $n$ can be embedded biholomorphically as a closed submanifold of $\mathbb{C}^{2 n+1}$ (cf. [Hö73]). Now the natural question is: Given a biinvariant Stein domain $D=G \exp _{G_{\mathbb{C}}}\left(i D_{h}\right)$ in $G_{\mathbb{C}}$, does there exist a $G \times G$ equivariant embedding into some complex Hilbert space $\mathcal{H}$ endowed with a unitary $G \times G$-action. In this paper we show that under quite natural assumptions the answer is affirmative. More concretly, if $\operatorname{Ad}(G)$ is closed in $\operatorname{Aut}(\mathfrak{g})$, the center $Z(G)$ is compact and the convex domain $D_{h}$ is pointed, then there exists a positive definite biinvariant holomorphic kernel $K$ on $D$, such that the map

$$
e_{K}: D \rightarrow \mathcal{H}_{K}^{*} \backslash\{0\}, \quad z \mapsto K_{z}
$$

defines a $G \times G$-equivariant closed embedding. Here $\mathcal{H}_{K}$ denotes the reproducing kernel Hilbert space and $K_{z}: \mathcal{H}_{K} \rightarrow \mathbb{C}, f \mapsto f(z)$ the point evaluations corresponding to $K$. 
Our method to construct such a kernel $K$ is to sum up kernels $K^{\lambda}$ associated to unitary highest weight representations $\left(\pi_{\lambda}, \mathcal{H}_{\lambda}\right)$ of $G$ over a certain lattice $\Gamma \subseteq i \mathfrak{t}^{*}$, where $\mathfrak{t}$ denotes a compactly embedded Cartan subalgebra of $\mathfrak{g}$. More precisely, we set

$$
K=\sum_{\lambda \in \Gamma}\|\lambda\|^{N} K^{\lambda}
$$

with $\|\cdot\|$ denoting a norm on $i \mathfrak{t}^{*}$ and $N \in \mathbb{N}$. These kernels $K$ have the important property of tending to infinity at the boundary of $D$, i.e.,

$$
\lim _{z \rightarrow \partial D} K(z, z)=\infty
$$

a result which is crucial for veryfying the closedness of the map $e_{K}$.

We think that our results are a little bit surprising and we do not really understand what is actually going on. For instance, what is the reason for that one has to exclude zero in $\mathcal{H}_{K}^{*}$ to achieve the closedness of the map $e_{K}$, or, is it possible to find an equivariant closed holomorphic embedding $D \rightarrow E$ into a complex topological vector space endowed with a continuous $G \times G$-action. We hope that our results give rise to a further discussion leading to a better understanding of these phenomena.

\section{The boundary behaviour of bi-invariant kernels.}

In this first section we characterize the boundary behaviour of biinvariant holomorphic positive definite kernels on a bi-invariant domain $D=$ $G \operatorname{Exp}\left(i D_{h}\right)$ by means of the boundary behaviour on the abelian submanifold $D_{T}:=T \operatorname{Exp}\left(i\left(D_{h} \cap \mathfrak{t}\right)\right)$. If the convex invariant set $D_{h} \subseteq \mathfrak{g}$ is a pointed cone, we show that $\lim _{z \rightarrow \partial D} K(z, z)=\infty$ if and only if $\lim _{z \rightarrow \partial D_{T}} K(z, z)=$ $\infty$. As abelian domains are comparable easily to deal with contrary to the highly non-commutative bi-invariant domains $D$, this result allows us in the sequel to make quite explicit computations.

Definition I.1. Let $V$ be a finite dimensional real vector space and $V^{*}$ its dual.

(a) For each subset $E \subseteq V$ we define its dual cone by $E^{\star}:=\{\alpha \in$ $\left.V^{*}:(\forall x \in E) \alpha(x) \geq 0\right\}$. We note that $E^{\star}$ is a convex closed subcone of $V^{*}$.

(b) For a convex subset $E \subseteq V$ we set

$H(E):=\{x \in V: x+E=E\}, \quad$ and $\quad \lim E:=\{x \in V: x+E \subseteq E\}$.

We call $H(E)$ the edge and $\lim E$ the limit cone of $E$. Note that $H(E)$ is a vector space, $H(E)=H(\bar{E})$ if $E$ is open and that $\lim E$ is a convex cone in $V$.

(c) A convex set $E$ is called pointed if it contains no affine lines. Note that if $E$ is open or closed then $E$ is pointed if and only if its edge is zero. 
Definition I.2. Let $\mathfrak{g}$ be a finite dimensional Lie algebra over $\mathbb{R}$.

(a) An element $X \in \mathfrak{g}$ is called elliptic if ad $X$ operates semisimply with purely imaginary spectrum. A convex cone $W \subseteq \mathfrak{g}$ is said to be elliptic if $W^{0} \neq \emptyset$ and all $X \in W^{0}$ are elliptic.

(b) For a subalgebra $\mathfrak{a} \subseteq \mathfrak{g}$ we write $\operatorname{Inn}(\mathfrak{a}):=\left\langle e^{\text {ad } \mathfrak{a}}\right\rangle \subseteq \operatorname{Aut}(\mathfrak{g})$ for the corresponding group of inner automorphisms. A subalgebra $\mathfrak{a} \subseteq \mathfrak{g}$ is said to be compactly embedded if $\operatorname{Inn}(\mathfrak{a})$ is relatively compact in $\operatorname{Aut}(\mathfrak{g})$.

(c) Let $\mathfrak{t} \subseteq \mathfrak{g}$ be a compactly embedded Cartan subalgebra and recall that there exists a unique maximal compactly embedded subalgebra $\mathfrak{k}$ containing $\mathfrak{t}$ (cf. [HHL89, A.2.40]).

(d) Associated to the Cartan subalgebra $\mathfrak{t}_{\mathbb{C}}$ in the complexification $\mathfrak{g}_{\mathbb{C}}$ is a root decomposition as follows. For a linear functional $\alpha \in \mathfrak{t}_{\mathbb{C}}^{*}$ we set

$$
\mathfrak{g}_{\mathbb{C}}^{\alpha}:=\left\{X \in \mathfrak{g}_{\mathbb{C}}:\left(\forall Y \in \mathfrak{t}_{\mathbb{C}}\right)[Y, X]=\alpha(Y) X\right\}
$$

and write $\Delta:=\left\{\alpha \in \mathfrak{t}_{\mathbb{C}}^{*} \backslash\{0\}: \mathfrak{g}_{\mathbb{C}}^{\alpha} \neq\{0\}\right\}$ for the set of roots. Then $\mathfrak{g}_{\mathbb{C}}=$ $\mathfrak{t}_{\mathbb{C}} \oplus \bigoplus_{\alpha \in \Delta} \mathfrak{g}_{\mathbb{C}}^{\alpha}, \alpha(\mathfrak{t}) \subseteq i \mathbb{R}$ for all $\alpha \in \Delta$ and $\overline{\mathfrak{g}_{\mathbb{C}}^{\alpha}}=\mathfrak{g}_{\mathbb{C}}^{-\alpha}$, where $X \rightarrow \bar{X}$ denotes complex conjugation on $\mathfrak{g}_{\mathbb{C}}$ with respect to $\mathfrak{g}$.

(e) A root $\alpha$ is said to be compact if $\mathfrak{g}_{\mathbb{C}}^{\alpha} \subseteq \mathfrak{k}_{\mathbb{C}}$ and non-compact otherwise. We write $\Delta_{k}$ for the set of compact roots and $\Delta_{n}$ for the non-compact ones. If $\mathfrak{g}=\mathfrak{r} \rtimes \mathfrak{s}$ is a $\mathfrak{k}$-invariant Levi decomposition, then we set

$$
\Delta_{r}:=\left\{\alpha \in \Delta: \mathfrak{g}_{\mathbb{C}}^{\alpha} \subseteq \mathfrak{r}_{\mathbb{C}}\right\} \quad \text { and } \quad \Delta_{s}:=\left\{\alpha \in \Delta: \mathfrak{g}_{\mathbb{C}}^{\alpha} \subseteq \mathfrak{s}_{\mathbb{C}}\right\}
$$

and recall that $\Delta=\Delta_{r} \dot{U} \Delta_{s}$ (cf. [Ne99, Ch. V]).

(f) A positive system $\Delta^{+}$of roots is a subset of $\Delta$ for which there exists a regular element $X_{0} \in i \mathrm{t}^{*}$ with $\Delta^{+}:=\left\{\alpha \in \Delta: \alpha\left(X_{0}\right)>0\right\}$. A positive system is said to be $\mathfrak{k}$-adapted if the set $\Delta_{n}^{+}:=\Delta_{n} \cap \Delta^{+}$is invariant under the Weyl group $\mathcal{W}_{\mathfrak{k}}:=N_{\operatorname{Inn}(\mathfrak{k})}(\mathfrak{t}) / Z_{\operatorname{Inn}(\mathfrak{k})}(\mathfrak{t})$ acting on $\mathfrak{t}$. We recall from $[\mathrm{Ne} 99, \mathrm{Ch} . \mathrm{V}]$ that there exists a $\mathfrak{k}$-adapted positive system if and only if $\mathfrak{z} \mathfrak{g}(\mathfrak{z}(\mathfrak{k}))=\mathfrak{k}$. In this case we call $\mathfrak{g}$ quasihermitian. In this case it is easy to see that $\mathfrak{s}$ is quasihermitian too, and so all simple ideals of $\mathfrak{s}$ are either compact or hermitian.

(g) We associate to a positive system $\Delta^{+}$the convex cones

$$
C_{\min }:=\operatorname{cone}\left\{i\left[\overline{X_{\alpha}}, X_{\alpha}\right]: X_{\alpha} \in \mathfrak{g}_{\mathbb{C}}^{\alpha}, \alpha \in \Delta_{n}^{+}\right\},
$$

and $C_{\max }:=\left(i \Delta_{n}^{+}\right)^{\star}=\left\{X \in \mathfrak{t}:\left(\forall \alpha \in \Delta_{n}^{+}\right) i \alpha(X) \geq 0\right\}$. Note that both $C_{\min }$ and $C_{\max }$ are closed convex cones in $\mathfrak{t}$.

$(\mathrm{h})$ Write $p_{\mathfrak{t}}: \mathfrak{g} \rightarrow \mathfrak{t}$ for the orthogonal projection along $[\mathfrak{t}, \mathfrak{g}]$ and set $\mathcal{O}_{X}:=\operatorname{Inn}(\mathfrak{g}) \cdot X$ for the adjoint orbit through $X \in \mathfrak{g}$. We define the minimal and maximal cone associated to $\Delta^{+}$by

$$
\begin{aligned}
W_{\min }: & =\left\{X \in \mathfrak{g}: p_{\mathfrak{t}}\left(\mathcal{O}_{X}\right) \subseteq C_{\min }\right\} \quad \text { and } \\
W_{\max }: & =\left\{X \in \mathfrak{g}: p_{\mathfrak{t}}\left(\mathcal{O}_{X}\right) \subseteq C_{\max }\right\}
\end{aligned}
$$

and note that both cones are convex closed and $\operatorname{Inn}(\mathfrak{g})$-invariant. 
From now on we assume that $\mathfrak{g}$ contains a compactly embedded Cartan subalgebra $\mathfrak{t} \subseteq \mathfrak{g}$ and that there exists an elliptic cone $W \subseteq \mathfrak{g}$. Then there exists a $\mathfrak{k}$-adapted positive system $\Delta^{+}$such that

$$
C_{\min } \subseteq W \cap \mathfrak{t} \subseteq C_{\max }
$$

holds and $W_{\max }$ is an elliptic cone (cf. [Ne96b, Th. II.11]). Moreover, we have $W_{\min } \cap \mathfrak{t}=C_{\min }$ and $W_{\max } \cap \mathfrak{t}=C_{\max }$ (cf. [Ne97, Lemma I.1]).

Definition I.3. (a) Let $W \subseteq \mathfrak{g}$ be a closed elliptic cone. Let $\tilde{G}$, resp. $\tilde{G}_{\mathbb{C}}$, be the simply connected Lie groups associated to $\mathfrak{g}$, resp. $\mathfrak{g}_{\mathbb{C}}$, and set $G_{1}:=\langle\exp \mathfrak{g}\rangle \subseteq \tilde{G}_{\mathbb{C}}$. Then Lawson's Theorem (cf. [HiNe93, Th. 7.34, $35])$ says that the subset $\Gamma_{G_{1}}(W):=G_{1} \exp (i W)$ is a closed subsemigroup of $G_{\mathbb{C}}$ and the polar map

$$
G_{1} \times W \rightarrow \Gamma_{G_{1}}(W), \quad(g, X) \mapsto g \exp (i X)
$$

is a homeomorphism.

Now the universal covering semigroup $\Gamma_{\tilde{G}}(W):=\tilde{\Gamma}_{G_{1}}(W)$ has a similar structure. We can lift the exponential function exp: $\mathfrak{g}+i W \rightarrow \Gamma_{G_{1}}(W)$ to an exponential mapping Exp: $\mathfrak{g}+i W \rightarrow \Gamma_{\tilde{G}}(W)$ with $\operatorname{Exp}(0)=\mathbf{1}$ and thus obtain a polar map

$$
\tilde{G} \times W \rightarrow \Gamma_{\tilde{G}}(W), \quad(g, X) \mapsto g \operatorname{Exp}(i X)
$$

which is a homeomorphism.

If $G$ is a connected Lie group associated to $\mathfrak{g}$, then $\pi_{1}(G)$ is a discrete central subgroup of $\Gamma_{\tilde{G}}(W)$ and we obtain a covering homomorphism $\Gamma_{\tilde{G}}(W) \rightarrow \Gamma_{G}(W):=\Gamma_{\tilde{G}}(W) / \pi_{1}(G)$ (cf. [HiNe93, Ch. 3]). It is easy to see that there is also a polar map $G \times W \rightarrow \Gamma_{G}(W),(g, X) \mapsto g \operatorname{Exp}(i X)$ which is a homeomorphism. The semigroups of the type $\Gamma_{G}(W)$ are called complex Ol'shanskiu semigroups.

The subset $\Gamma_{G}\left(W^{0}\right) \subseteq \Gamma_{G}(W)$ is an open semigroup carrying a complex manifold structure such that semigroup multiplication is holomorphic. Moreover there is an involution on $\Gamma_{G}(W)$ given by

$$
\text { * }: \Gamma_{G}(W) \rightarrow \Gamma_{G}(W), s=g \operatorname{Exp}(i X) \mapsto s^{*}=\operatorname{Exp}(i X) g^{-1}
$$

which is antiholomorphic on $\Gamma_{G}\left(W^{0}\right)$ (cf. [HiNe93, Th. 9.15] for a proof of all that). Thus $\Gamma_{G}(W)$ is an involutive semigroup.

(b) A bi-invariant domain $D \subseteq \Gamma_{G}\left(W_{\max }^{0}\right)$ is an open connected $G \times G$ bi-invariant subset of $\Gamma_{G}\left(W_{\max }^{0}\right)$. Note that

$$
D=G \operatorname{Exp}\left(i D_{h}\right)=G \operatorname{Exp}(i \mathcal{D}) G,
$$

where $D_{h} \subseteq W_{\max }^{0}$ and $\mathcal{D}=D_{h} \cap \mathfrak{t}$. Recall that $D$ is a Stein manifold if and only if $D_{h}$ is convex (cf. [Ne98, Th. 6.1]). In this case $D$ is called a bi-invariant Stein domain. We call $D$ pointed if $D_{h}$ is pointed in $\mathfrak{g}$. The 
boundary of a left $G$-invariant subset $E=G \operatorname{Exp}\left(i E_{h}\right) \subseteq \Gamma_{G}\left(W_{\max }\right)$ is defined as $\partial E:=G \operatorname{Exp}\left(i \partial E_{h}\right)$. Note that $\partial D=\bar{D} \backslash D$ for every bi-invariant domain $D$, where the closure $\bar{D}$ is taken in $\Gamma_{G}\left(W_{\max }\right)$.

Lemma I.4. Let $W \subseteq \mathfrak{g}$ be an invariant elliptic pointed convex cone and set $C:=W \cap \mathfrak{t}$. Let $\left(X_{n}\right)_{n \in \mathbb{N}}$ be a sequence in $W^{0}$ converging to $X \in \partial W$. Then there exists a subsequence $\left(X_{n_{k}}\right)_{k \in N}$ and a sequence $\left(Y_{n_{k}}\right)_{k \in \mathbb{N}}$ in $C^{0}$ with $Y_{n_{k}} \in \operatorname{Inn}(\mathfrak{g}) . X_{n_{k}}$ and $Y_{n_{k}} \rightarrow Y \in \partial C$.

Proof. W.l.o.g. we may assume that $W$ is closed. According to $[\mathbf{H i N e 9 3 ,}$ Th. 7.27], we can reconstruct $W^{0}$ from $C^{0}$, i.e., we have $W^{0}=\operatorname{Inn}(\mathfrak{g}) . C^{0}$. In particular, we find a sequence $\left(Y_{n}\right)_{n \in \mathbb{N}}$ in $C^{0}$ and a sequence $\left(g_{n}\right)_{n \in \mathbb{N}}$ in $\operatorname{Inn}(\mathfrak{g})$ such that $g_{n} \cdot X_{n}=Y_{n}$. We claim that $\left(Y_{n}\right)_{n \in \mathbb{N}}$ is bounded.

The Convexity Theorem for Adjoint Orbits (cf. [KrNe96, Th. VIII.9]) implies that

$$
p_{\mathfrak{t}}\left(X_{n}\right) \in \operatorname{conv}\left(\mathcal{W}_{\mathfrak{k}} \cdot Y_{n}\right)+C_{\min } \subseteq C
$$

for all $n \in \mathbb{N}$.

As $C$ is pointed, a sequence $\left(Z_{n}\right)_{n \in \mathbb{N}}$ in $C$ is unbounded if and only if $\varlimsup_{n \rightarrow \infty} \alpha\left(Z_{n}\right)=\infty$ holds for one $\alpha \in \operatorname{int} C^{\star}$. Thus if $\left(Y_{n}\right)_{n \in \mathbb{N}}$ is unbounded, then (1.1) together with the invariance of $C$ under $\mathcal{W}_{\mathfrak{k}}$ implies that $\left(p_{\mathfrak{t}}\left(X_{n}\right)\right)_{n \in \mathbb{N}}$ is unbounded. But this contradicts the fact that $\left(p_{\mathfrak{t}}\left(X_{n}\right)\right)_{n \in \mathbb{N}}$ being a continuous image of a Cauchy sequence is bounded, proving the claim.

Let now $\left(Y_{n_{k}}\right)_{k \in \mathbb{N}}$ be a convergent subsequence of $\left(Y_{n}\right)_{n \in \mathbb{N}}$ and $Y=$ $\lim _{k \rightarrow \infty} Y_{n_{k}}$ the corresponding limit in $C$. It remains to show that $Y \in \partial C$. To obtain a contradiction we assume that $Y \in C^{0}$.

We write $\mathrm{Sl}(W)$ for the special automorphism group of the cone $W$ and note that $\operatorname{Inn}(\mathfrak{g}) \subseteq \mathrm{Sl}(W)$ (cf. [HiNe93, Prop. 7.3(v)]). Then [HiNe93, Prop. 1.11] implies that there exists a convergent subsequence of $\left(g_{n_{k}}\right)_{k \in \mathbb{N}}$ in $\operatorname{Sl}(W)$ which we also denote by $\left(g_{n_{k}}\right)_{k \in \mathbb{N}}$. Write $g$ for the corresponding limit. Then

$$
X=\lim _{k \rightarrow \infty} g_{n_{k}}^{-1} \cdot Y_{n_{k}}=g^{-1} \cdot Y .
$$

Since $\operatorname{Sl}(W) \cdot W^{0}=W^{0}$ and $Y \in W^{0}$, this implies that $X \in W^{0}$; a contradiction, concluding the proof of the lemma.

Definition I.5. Let $M$ be a complex manifold and $\operatorname{Hol}(M)$ denote the space of holomorphic functions on $M$. We write $\bar{M}$ for $M$ equipped with the opposite complex structure.

(a) A function $K \in \operatorname{Hol}(M \times \bar{M})$ is called a holomorphic positive definite kernel if for every sequence $z_{1}, \ldots, z_{n}$ in $M$ the matrix $\left(K\left(z_{i}, z_{j}\right)\right)_{i, j}$ is positive semi-definite. We write $\mathcal{P}\left(M^{2}\right)$ for the convex cone of all holomorphic positive definite kernels on $M$. Note that every $K \in \mathcal{P}\left(M^{2}\right)$ satsifies the 
inequality

$$
(\forall z, w \in M)|K(z, w)| \leq \sqrt{K(z, z)} \sqrt{K(w, w)} .
$$

Recall that $K \in \mathcal{P}\left(M^{2}\right)$ if and only if there exists a Hilbert space $\mathcal{H} \subseteq$ $\operatorname{Hol}(M)$ with continuous point evaluations $K_{z}: \mathcal{H} \rightarrow \mathbb{C}, f \mapsto f(z)$ such that $K(z, w)=\left\langle K_{w}, K_{z}\right\rangle$ holds for all $(z, w) \in M \times M$ (cf. [Ne99, Ch. II]). In this case we also write $\mathcal{H}_{K}$ instead of $\mathcal{H}$ and refer to $\mathcal{H}_{K}$ as the reproducing kernel Hilbert space corresponding to $K$.

(b) An involutive semigroup is a semigroup $S$ together with an involutive antiautomorphism ${ }^{*}: S \rightarrow S$, i.e., $\left(s^{*}\right)^{*}=s$ and $(s t)^{*}=t^{*} s^{*}$ holds for all $s, t \in S$.

A mapping $\alpha: S \rightarrow \mathbb{R}^{+}$is called an absolute value if $\alpha\left(s^{*}\right)=\alpha(s)$ and $\alpha(s t) \leq \alpha(s) \alpha(t)$ hold for all $s, t \in S$. We denote by $\mathcal{A}(S)$ the collection of all absolute values on $S$.

(c) Let $S$ be an involutive semigroup acting on $M$ from the left by holomorphic mappings. A positive definite kernel $K$ is said to be $S$-invariant if $K(s . z, w)=K\left(z, s^{*} . w\right)$ holds for all $s \in S, z, w \in M$. We write $\mathcal{P}_{S}\left(M^{2}\right)$ for the subcone of $\mathcal{P}\left(M^{2}\right)$ of all $S$-invariant elements.

(d) An $S$-invariant positve definite kernel $K \in \mathcal{P}_{S}\left(M^{2}\right)$ is called $\alpha$-bounded for some $\alpha \in \mathcal{A}(S)$ if

$$
K(s . z, s . z) \leq \alpha(s) K(z, z)
$$

holds for all $z \in M, s \in S$. The set of all $\alpha$-bounded positve definite kernels is denoted by $\mathcal{P}_{S}\left(M^{2}, \alpha\right)$. Note that each $K \in \mathcal{P}_{S}\left(M^{2}, \alpha\right)$ gives rise to an involutive representation of $S$ given by

$$
\pi_{K}: S \rightarrow B\left(\mathcal{H}_{K}\right), \quad\left(\pi_{K}(s) . f\right)(z)=f\left(s^{*} . z\right),
$$

i.e., $\left(\pi_{K}, \mathcal{H}_{K}\right)$ is a representation of $S$ satisfying $\pi_{K}\left(s^{*}\right)=\pi_{K}(s)^{*}$ for all $s \in S$ (cf. [Ne99, Ch. II]).

We equip $G \times G$ with the involution $\left(g_{1}, g_{2}\right)^{*}=\left(g_{1}^{-1}, g_{2}^{-1}\right)$ for $g_{1}, g_{2} \in G$. Note that every $K \in \mathcal{P}_{G \times G}$ is trivially $\alpha$-bounded with $\alpha=\mathbf{1}$, and that

$$
\pi_{K}: G \times G \rightarrow U\left(\mathcal{H}_{K}\right), \quad\left(\pi_{K}\left(g_{1}, g_{2}\right) . f\right)(z)=f\left(g_{1}^{-1} z g_{2}\right)
$$

is a unitary representation of $G \times G(\mathrm{cf}$. [Kr97, Lemma III.6]).

Lemma I.6. Let $V$ be a finite dimensional real vector space, $V^{\sharp}:=V \oplus \mathbb{R}$ and $E \subseteq V$ a convex subset. Set $E^{\sharp}:=\mathbb{R}^{+}(E \times\{1\})$. Then $E^{\sharp}$ is a convex subcone of $V^{\sharp}$ and the following assertions hold:

(i) $E$ is closed in $E^{\sharp}$.

(ii) $E$ is pointed if and only if $E^{\sharp}$ is pointed.

(iii) If $E$ is open or closed, then $\left.\partial E^{\sharp}=\right] 0, \infty[.(\partial E \times\{1\}) \cup(\lim E \times\{0\})$.

Proof. (i) This is clear. 
(ii) If $E^{\sharp}$ is not pointed, then there exists a non-zero element $y=(x, r) \in$ $V^{\sharp}$ such that $\mathbb{R} y \subseteq E^{\sharp}$. In view of $E^{\sharp}=\mathbb{R}^{+}(E \times\{1\})$, we must have $r=0$. Now $\mathbb{R} y+E^{\sharp}=E^{\sharp}$ implies that $\mathbb{R} x+E=E$, i.e., $E$ is not pointed.

Conversely, if $E$ is not pointed, then there exists a non-zero element $x \in V$ such that $\mathbb{R} x \subseteq H(E)$. Then $\mathbb{R}(x, 0) \subseteq H\left(E^{\sharp}\right)$, i.e., $C$ is not pointed.

(iii) Note that $] 0, \infty\left[\left(E^{0} \times\{1\}\right)\right.$ is open and that $] 0, \infty[(\bar{E} \times\{1\})$ is closed in $V^{\sharp} \backslash(V \times\{0\})$. Hence $\left.\partial E^{\sharp} \cap V^{\sharp} \backslash(V \times\{0\})=\right] 0, \infty[(\partial E \times\{1\})$. By the definition of $E^{\sharp}$ we have $(x, 0) \in \partial E^{\sharp}$ if and only if there exists a sequence of positive real numbers $\left(\lambda_{n}\right)_{n \in \mathbb{N}}$ and a sequence $\left(x_{n}\right)_{n \in \mathbb{N}}$ such that $\lim _{n \rightarrow \infty} \lambda_{n}=0$ and $\lim _{n \rightarrow \infty} \lambda_{n} x_{n}=x$. In view of [Ne99, Prop. III.1.5(iii)], this means that $x \in \lim E$, concluding the proof of (iii).

Lemma I.7. Let $V$ be a finite dimensional real vector space and $E \subseteq V$ a convex set. Further let $V_{1}:=V / H(E)$, denote $q: V \rightarrow V_{1}$ the corresponding quotient homomorphism and set $E_{1}:=q(E)$. Then we have $q(\partial E)=\partial E_{1}$.

Proof. As $E+H(E)=E$ it follows that $E^{0}+H(E)=E^{0}$ and $\bar{E}+H(E)=$ $H(E)$. Thus $q(\bar{E})=\bar{E}_{1}$, and $q\left(E^{0}\right)=E_{1}^{0}$ since $q$ is an open mapping. This proves the lemma.

Proposition I.8. Let $\mathfrak{g}^{\sharp}=\mathfrak{g} \oplus \mathbb{R}, G^{\sharp}=G \times \mathbb{R}$, and

$$
D^{\sharp}:=\Gamma_{G^{\sharp}}\left(D_{h}^{\sharp}\right) \subseteq \Gamma_{G^{\sharp}}\left(W_{\max }^{0} \oplus \mathbb{R}\right) \cong \Gamma_{G}\left(W_{\max }^{0}\right) \oplus \mathbb{C} .
$$

(i) The map $j: D \rightarrow D^{\sharp}, s \mapsto(s, i)$ is a $G \times G$-equivariant holomorphic closed embedding inducing a map

$$
\mathcal{P}_{G^{\sharp} \times G^{\sharp}}\left(D^{\sharp^{2}}\right) \rightarrow \mathcal{P}_{G \times G}\left(D^{2}\right), \quad K^{\sharp} \mapsto K:=K^{\sharp} \circ j .
$$

(ii) Let $K \in \mathcal{P}_{G \times G}\left(D^{2}\right)$. Then the following statements are equivalent:

(a) $\lim _{z \rightarrow \partial D} K(z, z)=\infty$.

(b) $\lim _{X \rightarrow \partial D_{h}} K(\operatorname{Exp}(i X), \operatorname{Exp}(i X))=\infty$. Moreover, if $K=K^{\sharp} \circ j$ with some $K^{\sharp} \in \mathcal{P}_{G^{\sharp} \times G^{\sharp}}\left(D^{\sharp^{2}}\right)$, then (a)-(b) are implied by

(c) $\lim _{X \rightarrow \partial D_{h}^{\sharp}} K^{\sharp}(\operatorname{Exp}(i X), \operatorname{Exp}(i X))=\infty$.

(iii) Let $\mathfrak{a}:=H\left(D_{h}\right)$ be the edge of $D_{h}, \mathfrak{g}_{1}:=\mathfrak{g} / \mathfrak{a}$ and $q: \mathfrak{g} \rightarrow \mathfrak{g}_{1}$ the corresponding quotient morphism. Further let $A:=\langle\exp (\mathfrak{a})\rangle, G_{1}:=$ $G / A$ and $D_{1}:=G_{1} \operatorname{Exp}\left(i D_{h, 1}\right)$. Then the quotient morphism $\tilde{q}: D \rightarrow$ $D_{1}$ induced by $q$ gives rise to an injection

$$
\tilde{q}^{*}: \mathcal{P}_{G_{1} \times G_{1}}\left(D_{1}^{2}\right) \rightarrow \mathcal{P}_{G \times G}\left(D^{2}\right), \quad K_{1} \mapsto K:=K_{1} \circ \tilde{q}
$$

and the following statements are equivalent:

(a) $\lim _{X \rightarrow \partial D_{h}} K(\operatorname{Exp}(i X), \operatorname{Exp}(i X))=\infty$.

(b) $\lim _{X \rightarrow \partial D_{h, 1}} K_{1}(\operatorname{Exp}(i X), \operatorname{Exp}(i X))=\infty$.

(c) $\lim _{X \rightarrow \partial \mathcal{D}_{1}} K_{1}(\operatorname{Exp}(i X), \operatorname{Exp}(i X))=\infty$. 
Proof. (i) Obviously, $j$ is holomorphic and $G \times G$-equivariant. Further the closedness of $j$ follows from Lemma I.6(i) together with the Polar Decomposition of a complex Ol'shanskil semigroup. The second assertion is clear.

(ii) $(\mathrm{a}) \Rightarrow(\mathrm{b})$ is obvious.

(b) $\Rightarrow($ a): This follows from the biinvariance of $K$.

The remaining statement follows from Lemma I.6(iii).

(iii) (a) $\Longleftrightarrow$ (b): In view of $q\left(\partial D_{h}\right)=\partial D_{h, 1}$ (cf. Lemma I.7), this follows from the equivalence of (a) and (b) in (ii).

(b) $\Rightarrow(\mathrm{c})$ is clear.

$(c) \Rightarrow(b)$ follows from the $\operatorname{Inn}(\mathfrak{g})$-invariance of the map

$$
D_{h, 1} \rightarrow \mathbb{R}^{+}, \quad X \mapsto K_{1}(\operatorname{Exp}(i X), \operatorname{Exp}(i X))
$$

together with Lemma I.4.

\section{A kernel tending to infinity at the boundary.}

In this section we construct a kernel $K \in \mathcal{P}_{G \times G}\left(D^{2}\right)$ tending to infinity at the boundary. In view of Proposition I.8, this reduces to the case where $D=\Gamma_{G}(W)$ is a pointed complex Ol'shanskiu semigroup. But Proposition I.8(iii) tells us even more: We only have to check that $\lim _{X \mapsto \partial C} K(\operatorname{Exp}(i X)$, $\operatorname{Exp}(i X))=\infty$. To start out we need some notation concerning highest weight representations and their associated characters.

Definition II.1. Let $\Delta^{+}$be a positive system.

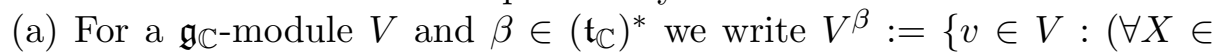
$\left.\left.\mathfrak{t}_{\mathbb{C}}\right) X . v=\beta(X) v\right\}$ for the weight space of weight $\beta$ and $\mathcal{P}_{V}=\left\{\beta: V^{\beta} \neq\{0\}\right\}$ for the set of weights of $V$.

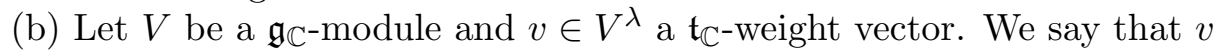
is a primitive element of $V$ (with respect to $\Delta^{+}$) if $\mathfrak{g}_{\mathbb{C}}^{\alpha} \cdot v=\{0\}$ holds for all $\alpha \in \Delta^{+}$.

(c) A $\mathfrak{g}_{\mathbb{C}}$-module $V$ is called a highest weight module with highest weight $\lambda$ (with respect to $\Delta^{+}$) if it is generated by a primitive element of weight $\lambda$.

(d) Let $\lambda \in i \mathrm{t}^{*}$ be dominant integral w.r.t. $\Delta_{k}^{+}$and $F(\lambda)$ the corresponding highest weight module for $\mathfrak{k}_{\mathbb{C}}$. Assume that $\Delta^{+}$is $\mathfrak{k}$-adapted and set $\mathfrak{p}^{ \pm}=$ $\bigoplus_{\alpha \in \Delta_{n}^{ \pm}} \mathfrak{g}_{\mathbb{C}}^{\alpha}$. We define the generalized Verma module by

$$
N(\lambda):=\mathcal{U}\left(\mathfrak{g}_{\mathbb{C}}\right) \otimes_{\mathcal{U}\left(\mathfrak{k}_{\mathbb{C}}+\mathfrak{p}^{+}\right)} F(\lambda) .
$$

Note that $N(\lambda)$ is a highest weight module for $\mathcal{U}\left(\mathfrak{g}_{\mathbb{C}}\right)$ with highest weight $\lambda$. We denote by $L(\lambda)$ the unique irreducible quotient of $N(\lambda)$.

(e) Let $G$ be a connected Lie group with Lie algebra $\mathfrak{g}$. We write $K$ for the analytic subgroup of $G$ corresponding to k. Let $(\pi, \mathcal{H})$ be a unitary representation of $G$. A vector $v \in \mathcal{H}$ is called $K$-finite if it is contained in 
a finite dimensional $K$-invariant subspace. We write $\mathcal{H}^{K, \omega}$ for the space of analytic $K$-finite vectors.

(f) An irreducible unitary representation $(\pi, \mathcal{H})$ of $G$ is called a highest weight representation w.r.t. $\Delta^{+}$with highest weight $\lambda \in i \mathrm{t}^{*}$ if $\mathcal{H}^{K, \omega}$ is a highest weight module for $\mathfrak{g}_{\mathbb{C}}$ w.r.t. $\Delta^{+}$and highest weight $\lambda$. We say that the irreducible highest weight module $L(\lambda)$ is unitarizable if there exists a unitary highest weight representation $\left(\pi_{\lambda}, \mathcal{H}_{\lambda}\right)$ of $\tilde{G}$ with $\mathcal{H}_{\lambda}^{K, \omega} \cong L(\lambda)$ as

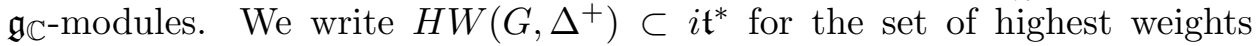
corresponding to unitary highest weight representations of $G$ w.r.t. $\Delta^{+}$and write $H W\left(\Delta^{+}\right):=H W\left(\tilde{G}, \Delta^{+}\right)$for the set of all highest weights w.r.t. $\Delta^{+}$ which correspond to a unitarizable $L(\lambda)$.

(g) Let $\lambda \in H W\left(\Delta^{+}\right)$. We call $\lambda$ singular if the natural map $N(\lambda) \rightarrow$ $L(\lambda)$ has a non-trivial kernel and non-singular otherwise.

For each unitary representation $(\pi, \mathcal{H})$ of $G$ we write $\left(\pi^{*}, \mathcal{H}^{*}\right)$ for the corresponding dual representation. Let $B_{2}(\mathcal{H})$ be the space of Hilbert Schmidt operators on $\mathcal{H}$. We define a representation of $G \times G$ on $B_{2}(\mathcal{H})$ by

$$
\pi^{c}: G \times G \rightarrow U\left(B_{2}(\mathcal{H})\right), \quad \pi^{c}\left(g_{1}, g_{2}\right) . A:=\pi\left(g_{2}\right) A \pi\left(g_{1}\right)^{*} .
$$

Note that there is a canonical isomorphism between $\left(\pi^{*} \otimes \pi, \mathcal{H}^{*} \widehat{\otimes} \mathcal{H}\right)$ and $\left(\pi^{c}, B_{2}(\mathcal{H})\right)$.

Now we fix a positive system $\Delta^{+}$associated to $W_{\max }$.

Recall from $[\mathrm{HiNe}$ 96, Th. 3.6, Th. B] that each highest weight representation $\left(\pi_{\lambda}, \mathcal{H}_{\lambda}\right)$ of $G$ extends to an holomorphic representation of $\Gamma_{G}\left(W_{\max }\right)$ denoted by the same symbol. Moreover all operators $\pi_{\lambda}(s), s \in \Gamma_{G}\left(W_{\max }^{0}\right)$, are of trace class (cf. [Ne94, Th. III.8]), so that $\Theta_{\lambda}(s):=\operatorname{tr} \pi_{\lambda}(s)$ makes sense for all $s \in \Gamma_{G}\left(W_{\max }^{0}\right)$. We call $\Theta_{\lambda}$ the character of $\left(\pi_{\lambda}, \mathcal{H}_{\lambda}\right)$ and note that $\Theta_{\lambda}$ is holomorphic on $\Gamma_{G}\left(W_{\max }^{0}\right)$ (cf. [Ne94, Th. IV.11]).

Associated to a $\mathfrak{k}$-adapted positive system, we define the function

$$
\phi: i C_{\max }^{0} \rightarrow \mathbb{R}^{+}, \quad X \mapsto \frac{1}{\prod_{\alpha \in \Delta_{n}^{+}}\left(1-e^{-\alpha(X)}\right)^{m_{\alpha}}},
$$

where $m_{\alpha}:=\operatorname{dim}_{\mathbb{C}} \mathfrak{g}_{\mathbb{C}}^{\alpha}$ for all $\alpha \in \Delta$.

Lemma II.2. Let $\lambda \in H W\left(G, \Delta^{+}\right)$be non-singular and $\left(\pi_{\lambda}, \mathcal{H}_{\lambda}\right)$ an associated highest weight representation of $G$. If $\Theta_{\lambda}^{K}$ denotes the character of $F(\lambda)$, then

$$
\Theta_{\lambda}(\operatorname{Exp} X)=\phi(X) \Theta_{\lambda}^{K}(\operatorname{Exp}(X))
$$

for all $X \in i C_{\max }^{0}$.

Proof. [Kr97, Lemma IV.8(i)].

Proposition II.3. Let $K$ be a connected Lie group with compact Lie algebra $\mathfrak{k}$. Let $\mathfrak{t}$ be a Cartan subalgebra of $\mathfrak{k}, \Delta_{k}^{+}$be a positive system of roots and $\lambda \in i \mathbf{t}^{*}$ be a dominant analytically integral element. Further let $\Theta_{\lambda}^{K}$ denote 
the character of the holomorphic representation $\left(\pi_{\lambda}^{K}, F(\lambda)\right)$ of $K_{\mathbb{C}}$ and set $d_{\lambda}:=\operatorname{dim} F(\lambda)$.

(i) $(\forall X \in i \mathfrak{t}) e^{\lambda(X)} \leq \Theta_{\lambda}^{K}\left(\exp _{K_{\mathbb{C}}}(X)\right) \leq d_{\lambda} \sup _{w \in \mathcal{W}_{k}} e^{\lambda(w \cdot X)}$.

(ii) If $\|\cdot\|$ denotes a norm on $i^{*}$, then there exists a constant $c>0$ and an element $n \in \mathbb{N}$ such that

$$
d_{\lambda} \leq c\|\lambda\|^{n}+c
$$

holds for all integral elements $\lambda$.

Proof. (i) Let $\left\{v_{j}: 1 \leq j \leq d_{\lambda}\right\}$ be an orthonormal basis of weight vectors of $F(\lambda)$. For each $j$ let $\alpha_{j}$ be the weight corresponding to $v_{j}$. Then we have for all $X \in \mathfrak{t}_{\mathbb{C}}$ that

$$
\Theta_{\lambda}^{K}\left(\exp _{K_{\mathbb{C}}}(X)\right)=\sum_{j=1}^{d_{\lambda}}\left\langle\pi_{\lambda}^{K}\left(\exp _{K_{\mathbb{C}}}(X)\right) \cdot v_{j}, v_{j}\right\rangle=\sum_{j=1}^{d_{\lambda}} e^{\alpha_{j}(X)} .
$$

Since $\lambda=\alpha_{j}$ for some $1 \leq j \leq d_{\lambda}$, this proves the first inequality.

To prove the second inequality, we first observe that both $\Theta_{\lambda}^{K}\left(\exp _{K_{\mathbb{C}}}(X)\right)$ and $d_{\lambda} \sup _{w \in \mathcal{W}_{k}} e^{\lambda(w \cdot X)}$ considered as functions of $X \in i \mathrm{t}^{*}$ are invariant under the Weyl group $\mathcal{W}_{k}$. Thus we may assume that $X \in-\left(\Delta_{k}^{+}\right)^{\star}:=$ $\left\{Y \in i \mathrm{t}^{*}:\left(\forall \alpha \in \Delta_{k}^{+}\right) \alpha(X) \leq 0\right\}$. Since $\lambda(X)=\sup _{1 \leq j \leq d_{\lambda}} \alpha_{j}(X)$ whenever $X \in-\left(\Delta_{k}^{+}\right)^{\star},(2.1)$ implies that $\Theta_{\lambda}^{K}\left(\exp _{K_{\mathbb{C}}}(X)\right) \leq d_{\lambda} e^{\bar{\lambda}(X)}$, concluding the proof of (i).

(ii) This is a direct consequence of the Weyl Dimension Formula.

Corollary II.4. If $\lambda \in H W\left(G, \Delta^{+}\right)$is non-singular, then there exist a constant $c>0$ and $n \in \mathbb{N}$ such that

$$
e^{\lambda(X)} \leq \Theta_{\lambda}(\operatorname{Exp}(X)) \leq\left(c\|\lambda\|^{n}+c\right) \phi(X) \sup _{w \in \mathcal{W}_{k}} e^{\lambda(w \cdot X)}
$$

holds for all $X \in i C_{\max }^{0}$.

Proof. As $1=\inf _{X \in i C_{\max }^{0}} \phi(X)$, the corollary follows from Lemma II.2 and Proposition II.3.

Lemma II.5. Let $V$ be a finite dimensional real vector space, $C \subseteq V$ a convex cone with non-empty interior, $\Gamma \subseteq V$ a lattice and $Q \subseteq V$ a compact subset. Then

$$
\Gamma(C, Q):=\{\gamma \in \Gamma: \gamma \in C, \gamma+Q \subseteq C\}
$$

is an additive subsemigroup of $\Gamma, \overline{\mathbb{R}^{+} \Gamma(C, Q)}$ is a closed convex cone and the following equalities hold:

(i) $\overline{\mathbb{R}^{+} \Gamma(C, Q)}=\bar{C}$.

(ii) $\Gamma(C, Q)^{\star}=C^{\star}$. 
Proof. First we show that $\Gamma(C, Q)$ is an additive semigroup. Let $\gamma_{1}, \gamma_{2} \in$ $\Gamma(C, Q)$. Then $\gamma_{1}+\gamma_{2} \in C$ since $C$ is a convex cone and further we have for all $x \in Q$

$$
\gamma_{1}+\gamma_{2}+x=\gamma_{1}+\underbrace{\left(\gamma_{2}+x\right)}_{\in C} \in C+C=C,
$$

proving that $\Gamma(C, Q)$ is an additive semigroup.

It follows in particular that $\mathbb{Q}^{+} \Gamma(C, Q)$ is an additive semigroup and hence the same holds for $\overline{\mathbb{R}^{+} \Gamma(C, Q)}=\overline{\mathbb{Q}^{+} \Gamma(C, Q)}$. This proves that $\overline{\mathbb{R}^{+} \Gamma(C, Q)}$ is a closed convex cone.

(i) Since $\Gamma(C, Q) \subseteq C$ we obtain in particular that $\overline{\mathbb{R}^{+} \Gamma(C, Q)} \subseteq \bar{C}$.

To prove the converse inclusion, we assume that $\overline{\mathbb{R}^{+} \Gamma(C, Q)} \neq \bar{C}$. Then we find an open ball $B \subseteq V$ such that $B \subseteq C^{0} \backslash \overline{\mathbb{R}^{+} \Gamma(C, Q)}$. Since $C^{0} \backslash \overline{\mathbb{R}^{+} \Gamma(C, Q)}$ is an open cone, this implies in particular that

$$
(\forall \lambda>0) \lambda B \cap \Gamma(C, Q)=\emptyset .
$$

If $\lambda$ is sufficiently large, then we have $\lambda B \subseteq C-x$ for all $x \in Q$, because $B+\frac{1}{\lambda} x$ is contained in $C$ for sufficiently large $\lambda$ and $Q$ is compact. Let $\lambda_{0}>0$ such that $\lambda B \subseteq C-x$ for all $x \in Q$ and $\lambda>\lambda_{0}$.

In view of this and $\Gamma(C, Q)=\Gamma \cap C \cap \bigcap_{x \in Q}(C-x)$, (2.2) implies in particular that

$$
\left(\forall \lambda>\lambda_{0}\right) \Gamma \cap C \cap \lambda B=\Gamma(C, Q) \cap \lambda B=\emptyset ;
$$

a contradiction, concluding the proof of (i).

(ii) This follows from (i) and $\overline{\mathbb{R}^{+} \Gamma(C, Q)^{\star}}=\Gamma(C, Q)^{\star}$.

Let $\mathfrak{t}_{e}:=\{X \in \mathfrak{t}: \exp (X)=\mathbf{1}\}$ and note that $\mathfrak{t}_{e}$ is a lattice in $\operatorname{span}\left\{\mathfrak{t}_{e}\right\}$. Hence we find a lattice $\Gamma \in i \mathrm{t}^{*}$ which is contained in the set $\left\{\alpha \in i \mathrm{t}^{*}:(\forall X \in\right.$ $\left.\left.\mathfrak{t}_{e}\right) \alpha(X) \in 2 \pi i \mathbb{Z}\right\}$. From now on we fix a lattice $\Gamma \subseteq i \mathfrak{t}^{*}$ having this property.

Lemma II.6. Let $W \subseteq \mathfrak{g}$ be a pointed closed $\operatorname{Inn}(\mathfrak{g})$-invariant cone with non-empty interior, $\Delta^{+}$a positive system satisfying $C_{\min } \subseteq C=W \cap \mathfrak{t} \subseteq$ $C_{\max }$ and $\rho:=\frac{1}{2} \sum_{\alpha \in \Delta^{+}} m_{\alpha} \alpha$. Then

$$
\Gamma\left(i \operatorname{int} C^{\star}, 2 \rho\right):=\left\{\lambda \in \Gamma: \lambda \in i \operatorname{int} C^{\star}, \lambda+2 \rho \in i \operatorname{int} C^{\star}\right\}
$$

consists of non-singular $G$-analytically integral elements.

Proof. First note that $\Gamma$ consists of $G$-analytically integral elements, hence the same holds for the subset $\Gamma\left(i\right.$ int $\left.C^{\star}, 2 \rho\right)$. We claim that $\Gamma\left(i \operatorname{int} C^{\star}, 2 \rho\right) \subseteq$ $\Gamma\left(i\right.$ int $\left.C^{\star}, \rho\right)$. In fact, Lemma II.5 implies that $\Gamma\left(i\right.$ int $\left.C^{\star}, 2 \rho\right)$ is an additive semigroup, so that $\lambda \in \Gamma\left(i\right.$ int $\left.C^{\star}, 2 \rho\right)$ implies that $2 \lambda \in \Gamma\left(i\right.$ int $\left.C^{\star}, 2 \rho\right)$ which means that $2 \lambda+2 \rho \in i$ int $C^{\star}$ or equivalently $\lambda+\rho \in \Gamma\left(i\right.$ int $\left.C^{\star}, \rho\right)$. This proves the claim.

Further, $C_{\min } \subseteq C$ implies that $i$ int $C^{\star} \subseteq i$ int $C_{\text {min }}^{\star}$ and we therefore get

$$
\Gamma\left(i \operatorname{int} C^{\star}, 2 \rho\right) \subseteq\left\{\lambda \in \Gamma: \lambda+\rho \in i \operatorname{int} C_{\min }^{\star}\right\} .
$$


In view of $[\mathrm{Ne} 99, \mathrm{Ch} . \mathrm{IX}]$, this implies that all elements of $\Gamma\left(i \operatorname{int} C^{\star}, 2 \rho\right)$ are non-singular.

Lemma II.7. Let $V$ be a finite dimensional real vector space, $C \subseteq V$ a convex pointed cone with non-empty interior, $\Gamma \subseteq V^{*}$ a lattice and $Q \subseteq V^{*}$ a compact subset. We fix a norm $\|\cdot\|$ on $V^{*}$ with $\|\gamma\| \geq 1$ for all $\gamma \in \Gamma \backslash\{0\}$ and consider for each $N \in \mathbb{N}_{0}$ the mapping

$$
F^{N}: V \rightarrow \mathbb{R}^{+} \cup\{\infty\}, \quad x \mapsto \sum_{\gamma \in \Gamma\left(\operatorname{int} C^{\star}, Q\right)}\|\gamma\|^{N} e^{-\gamma(X)} .
$$

(i) For all $N \in \mathbb{N}_{0}$ the series defining $F^{N}$ converges compactly on $C^{0}$. In particular, $\left.F^{N}\right|_{C^{0}}$ is continuous.

(ii) For all $N \geq 1$ we have $\lim _{\substack{x \rightarrow O C \\ X \in C^{0}}} F^{N}(x)=\infty$.

Proof. (i) Since $C^{\star}$ is pointed we find for every $x \in C^{0}$ a constant $C_{x}>0$ such that $\|\alpha\| \leq C_{x} \alpha(x)$ holds for every $\alpha \in C^{\star}$. Thus we find for every compact subset $K \subseteq C^{0}$ a constant $C_{K}>0$ such that

$$
(\forall x \in K)\left(\forall \alpha \in C^{\star}\right)\|\alpha\| \leq C_{K} \alpha(x) .
$$

This in turn implies that

$$
\sup _{x \in K} \sum_{\gamma \in \Gamma\left(\operatorname{int} C^{\star}, Q\right)}\|\gamma\|^{N} e^{-\gamma(x)} \leq \sum_{\gamma \in \Gamma\left(\operatorname{int} C^{\star}, Q\right)}\|\gamma\|^{N} e^{-\frac{1}{C_{K}}\|\gamma\|}<\infty,
$$

proving (i).

(ii) As $\|\gamma\| \geq 1$ for all $\gamma \in \Gamma \backslash\{0\}$, we have $F^{1} \leq F^{N}$ for all $N \in \mathbb{N}$ and hence we only have to prove the assertion for $N=1$. Let $\left(x_{n}\right)_{n \in \mathbb{N}}$ be a sequence in $C^{0}$ converging to $x \in \partial C$. Then there exists an element $0 \neq \alpha \in \partial C^{\star}$ such that $\alpha(x)=0$. Choose $\alpha_{n} \in \mathbb{R}^{+} \alpha$ with $\alpha_{n}\left(x_{n}\right)=1$. We claim that $\lim _{n \rightarrow \infty}\left\|\alpha_{n}\right\|=\infty$. Indeed, otherwise we find a number $L>0$ and a subsequence $\left(\alpha_{n_{k}}\right)_{k \in \mathbb{N}}$ such that $\alpha_{n_{k}} \in[0, L] \alpha$. But then

$$
1=\alpha_{n_{k}}\left(x_{n_{k}}\right) \leq L \alpha\left(x_{n_{k}}\right) \rightarrow 0
$$

yields a contradiction and proves the claim.

Next we claim that there exists a constant $c>0$ and elements $\gamma_{n} \in$ $\Gamma\left(\right.$ int $\left.C^{\star}, Q\right)$ such that $\left\|\gamma_{n}-\alpha_{n}\right\|<c$ for all $n \in \mathbb{N}$. Let $r>0$ such that $B(\beta, r):=\left\{\mu \in V^{*}:\|\beta-\mu\| \leq r\right\}$ intersects $\Gamma$ for all $\beta \in V^{*}$. Now choose $\mu \in \operatorname{int} C^{\star}$ such that $B(\mu+t \alpha, r)+Q \subseteq \operatorname{int} C^{\star}$ holds for all $t \geq 0$. Then we find elements $\gamma_{n} \in \Gamma\left(\operatorname{int} C^{\star}, Q\right) \cap B\left(\mu+\alpha_{n}, r\right)$. These elements $\gamma_{n}$ satisfy $\left\|\gamma_{n}-\alpha_{n}\right\| \leq c$ with $c=r+\|\mu\|$, proving our second claim. 
Now we get

$$
\begin{aligned}
F_{1}\left(x_{n}\right) & =\sum_{\gamma \in \Gamma\left(\operatorname{int} C^{\star}, Q\right)}\|\gamma\| e^{-\gamma\left(x_{n}\right)} \geq\left\|\gamma_{n}\right\| e^{-\left(\gamma_{n}\left(x_{n}\right)-\alpha_{n}\left(x_{n}\right)\right)} e^{-\alpha_{n}\left(x_{n}\right)} \\
& \geq \frac{\left\|\gamma_{n}\right\|}{e} e^{-\left\|\gamma_{n}-\alpha_{n}\right\| \cdot\left\|x_{n}\right\|} \geq \frac{1}{e} e^{-c\left\|x_{n}\right\|}\left(\left\|\alpha_{n}\right\|-c\right),
\end{aligned}
$$

and so

$$
\lim _{n \rightarrow \infty} F_{1}\left(x_{n}\right) \geq \lim _{n \rightarrow \infty} \frac{1}{e} e^{-c\left\|x_{n}\right\|}\left(\left\|\alpha_{n}\right\|-c\right)=\infty .
$$

This proves (ii).

Theorem II.8. Let $S=\Gamma_{G}(W)$ be a pointed complex Ol'shanskiu semigroup. Then for all $N \in \mathbb{N}$ the prescription

$$
K^{N}: S^{0} \times S^{0} \rightarrow \mathbb{C}, \quad(z, w) \mapsto \sum_{\lambda \in \Gamma\left(i \operatorname{int} C^{\star}, 2 \rho\right)}\|\lambda\|^{N} \Theta_{\lambda}\left(z w^{*}\right)
$$

defines an element of $\mathcal{P}_{G \times G}\left(S^{0^{2}}\right)$ satisfying

$$
\lim _{z \mapsto \partial S^{0}} K^{N}(z, z)=\infty .
$$

Proof. First we show that $K^{N} \in \mathcal{P}_{G \times G}\left(S^{0^{2}}\right)$ for all $N \in \mathbb{N}$. Since all kernels

$$
K^{\lambda}: S^{0} \times S^{0} \rightarrow \mathbb{C}, \quad(z, w) \mapsto \Theta_{\lambda}\left(z w^{*}\right)
$$

belong to $\mathcal{P}_{G \times G}\left(S^{0^{2}}\right)$ (cf. [Ne94, Th. IV.11]), we only have to show that the series defining $K^{N}$ converges uniformly on compact subsets. In view of (1.2), a series of holomorphic positive definite kernels on a complex manifold converges compactly if and only if it converges uniformly on compact subsets on the diagonal. Therefore the bi-invariance of the kernels $K^{\lambda}$ together with the Polar Decomposition of $S^{0}$ imply that it suffices to prove the compact convergence of the series defining the function

$$
\Theta^{N}: i W^{0} \rightarrow \mathbb{R}^{+}, \quad X \mapsto \sum_{\lambda \in \Gamma\left(i \operatorname{int} C^{\star}, 2 \rho\right)}\|\lambda\|^{N} \Theta_{\lambda}(\operatorname{Exp}(X)) .
$$

If $C\left(W^{0}\right)^{G}$ denotes the $\operatorname{Ad}(G)$-invariant continuous functions on $W^{0}$ endowed with the topology of uniform convergence on compact subsets, then [Ne96b, Prop. III.6] entails that the restriction mapping $C\left(W^{0}\right)^{G} \rightarrow$ $C\left(C^{0}\right)^{\mathcal{W}_{\mathfrak{k}}}$ is an isomorphism of Fréchet spaces. Thus we have reduced the problem to showing the compact convergence of the series defining $\left.\Theta^{N}\right|_{C^{0}}$. In view of Corollary II.4, we therefore have to prove that the series defined by

$$
\left.F^{N}\right|_{i C^{0}}: i C^{0} \rightarrow \mathbb{R}^{+}, \quad X \mapsto \sum_{\lambda \in \Gamma\left(i \operatorname{int} C^{\star}, 2 \rho\right)}\|\lambda\|^{N} e^{\lambda(X)}
$$

converges compactly. But this is exactly the contents of Lemma II.7(i). 
In order to prove the second assertion, Proposition I.8(iii) implies that we only have to check that $\lim _{\substack{X \mapsto X^{0} \\ X \in C^{0}}} K^{N}(\operatorname{Exp}(i X), \operatorname{Exp}(i X))=\infty$. According to Corollary II.4, this follows from $\lim _{\substack { X \rightarrow C^{0} \\ \begin{subarray}{c}{X \\ \text { (n) }{ X \rightarrow C ^ { 0 } \\ \begin{subarray} { c } { X \\ \text { (n) } } }\end{subarray}} F^{N}(X)=\infty$ which is the contents of Lemma II.7(ii). This proves the theorem.

Corollary II.9. Let $D \subseteq \Gamma_{G}\left(W_{\max }^{0}\right)$ be a bi-invariant Stein domain. Then there exists a kernel $K \in \mathcal{P}_{G \times G}\left(D^{2}\right)$ such that

$$
\lim _{z \mapsto \partial D} K(z, z)=\infty .
$$

Proof. In view of Proposition I.8(ii), we may assume that $D_{h}=D_{h}^{\sharp}$ is a cone, and hence Proposition I.8(iii) implies that we even may assume that $D_{h}=D_{h, 1}^{\sharp}$ is a pointed cone. Now the corollary follows from Theorem II.8.

Remark II.10. One can modify $\Gamma$ a little bit without affecting the contents Theorem II.8 as follows. If one takes $\Gamma^{\prime}=\Gamma \cup F$, where $F \subseteq H W\left(G, \Delta^{+}\right)$ is a finite set of highest weights, then Theorem II.8 remains true with $\Gamma$ replaced by $\Gamma^{\prime}$.

\section{The equivariant embedding theorem.}

In this section we apply the results of Section II to construct a kernel $K \in$ $\mathcal{P}_{G \times G}\left(D^{2}\right)$ such that the map

$$
e_{K}: D \mapsto \mathcal{H}_{K}^{*} \backslash\{0\}, \quad z \mapsto K_{z}
$$

defines a $G \times G$-equivariant holomorphic embedding with closed image.

Proposition III.1. Let $M$ be a complex manifold, $S$ an involutive semigroup acting on $M$ by holomorphic maps and $K \in \mathcal{P}_{S}\left(M^{2}\right)$. Then

$$
e_{K}: M \rightarrow \mathcal{H}_{K}^{*}, \quad z \mapsto K_{z}
$$

is an $S$-equivariant holomorphic map.

Proof. From the $S$-invariance of $K$ it follows that

$$
e_{K}(s . z)=K_{s . z}=\pi_{K}(s) \cdot K_{z}=\pi_{K}(s) \cdot e_{K}(z)
$$

for all $s \in S$ and $z \in M$, proving the $S$-equivariance of the map $e_{K}$. It remains to show that $e_{K}$ is holomorphic. As $\mathcal{H}_{K}$ is a Hilbert space, $e_{K}$ is holomorphic if and only if the following conditions are satisfied:

(1) $e_{K}$ is locally bounded.

(2) There exists a total subset $T \subseteq \mathcal{H}_{K}$ such that the mappings $M \rightarrow$ $\mathbb{C}, z \mapsto K_{z}(f)=\left\langle f, K_{z}\right\rangle$ are holomorphic for all $f \in T$. 
If $Q \subseteq D$ is a compact subset, then we have

$$
\sup _{z \in Q}\left\|K_{z}\right\|^{2}=\sup _{z \in Q} K(z, z)<\infty,
$$

proving (1). Finally $T=\left\{K_{w}: w \in M\right\}$ is a total subset in $\mathcal{H}_{K}$ and $z \mapsto$ $K_{z}\left(K_{w}\right)=\left\langle K_{w}, K_{z}\right\rangle=K(z, w)$ is holomorphic because $K \in \operatorname{Hol}(M \times \bar{M})$. This proves (2) and concludes the proof of the lemma.

Corollary III.2. If $K \in \mathcal{P}_{G \times G}\left(D^{2}\right)$ is non-zero, then $K_{z} \neq 0$ for all $z \in D$ and the mapping

$$
e_{K}: D \rightarrow \mathcal{H}_{K}^{*} \backslash\{0\}, \quad z \mapsto K_{z}
$$

is $G \times G$-equivariant and holomorphic.

Proof. It follows from [Ne97, Lemma III.6] that $K_{z} \neq 0$ for all $z \in D$ and thus the map $e_{K}$ is well defined. Now the assertions follow from Proposition III.1.

Definition III.3. A connected Lie group is called a (CA)-Lie group if $\operatorname{Ad}(G) \subseteq \operatorname{Aut}(\mathfrak{g})$ is closed. Note that all connected reductive and nilpotent Lie groups are (CA)-Lie groups.

The next lemma is our key observation. The proof depends heavily on the special choice of the lattice $\Gamma\left(i\right.$ int $\left.C^{\star}, 2 \rho\right)$ and is a little bit tricky.

Lemma III.4. Let $S=\Gamma_{G}(W)$ be a pointed complex Ol'shanskiu semigroup and suppose that $G$ is a (CA)-Lie group. Let $K^{N}, N \in \mathbb{N}$, be as in Theorem II.8 and $\left(K_{s_{n}}^{N}\right)_{n \in \mathbb{N}}$ a convergent sequence in $\mathcal{H}_{K^{N}}$ with limit different from zero. Further let $Z$ denote the center of $G$.

(i) The set $\left\{s_{n} Z: n \in \mathbb{N}\right\}$ is relatively compact in $S^{0} / Z$.

(ii) If, in addition, $Z$ is compact, then $\left\{s_{n}: n \in \mathbb{N}\right\}$ is relatively compact in $S^{0}$.

Proof. (i) For simplicity, we write $K$ instead of $K^{N}$. Since $K=\sum_{\lambda}\|\lambda\|^{N} K^{\lambda}$ is a direct sum of positive definite kernels corresponding to inequivalent irreducible unitary representations of $G \times G$, it follows in particular that $\mathcal{H}_{K}=\widehat{\bigoplus}_{\lambda} \mathcal{H}_{K^{\lambda}}$ (cf. [Ne99, Th. I.11, Rem. I.12(a)]). Thus $\left(K_{s_{n}}\right)_{n \in \mathbb{N}}$ being a convergent sequence with non-zero limit implies in particular that all sequences $\left(K_{s_{n}}^{\lambda}\right)_{n \in \mathbb{N}}$ are convergent and at least one limit $f^{\lambda}=\lim _{n \rightarrow \infty} K_{s_{n}}^{\lambda}$ is different from zero.

Step 1: The set $\left\{s_{n} Z: n \in \mathbb{N}\right\}$ is relatively compact in $S / Z$.

Let $\lambda \in \Gamma\left(i\right.$ int $\left.C^{\star}, 2 \rho\right)$ be such that $\left(K_{s_{n}}^{\lambda}\right)_{n \in \mathbb{N}}$ converges with limit different from zero. Let

$$
\chi_{\lambda}: Z \rightarrow \mathrm{S}^{1}, \quad z=\exp (X) \mapsto e^{-\lambda(X)}
$$

and note that $\chi_{\lambda}$ is an element of $\widehat{Z}$. 
The Bergman space corresponding to the character $\chi_{\lambda}$ is defined as

$$
\begin{array}{r}
\mathcal{B}^{2}\left(S / Z, \chi_{\lambda}\right)=\left\{f \in \operatorname{Hol}\left(S^{0}\right):\left(\forall z \in Z, s \in S^{0}\right) f(s z)=\chi_{\lambda}(z)^{-1} f(s),\right. \\
\left.\|f\|_{2}^{2}:=\int_{S^{0} / Z}|f(s)|^{2} d \mu_{S^{0} / Z}(s Z)<\infty\right\},
\end{array}
$$

where $\mu_{S^{0} / Z}$ denotes the canonical left $S$-invariant measure on $S^{0} / Z$ (cf. $[$ Kr98, Sect. II] $)$. Recall from [Kr98, Prop. II.4, Th. IV.5] that $\mathcal{B}^{2}\left(S / Z, \chi_{\lambda}\right)$ is a closed subspace of the Hilbert space $L^{2}\left(S / Z, \chi_{\lambda}\right)$ and that there exists a positive constant $c>0$ such that the prescription

$$
\mathcal{H}_{K^{\lambda}} \rightarrow \mathcal{B}^{2}\left(S / Z, \chi_{\lambda}\right), \quad K_{z}^{\lambda} \mapsto c K_{z}^{\lambda}
$$

defines an $S \times S$-equivariant isometric embedding.

We obtain in particular that $\left(K_{s_{n}}^{\lambda}\right)_{n \in \mathbb{N}}$ is a convergent sequence in $\mathcal{B}^{2}\left(S / Z, \chi_{\lambda}\right)$ with limit $f^{\lambda} \neq 0$. To obtain a contradiction, we now assume that there exists a subsequence of $\left(s_{n} Z\right)_{n \in \mathbb{N}}$ leaving every compact subset of $S / Z$. To avoid further notation we denote this subsequence again by $\left(s_{n} Z\right)_{n \in \mathbb{N}}$. Note that $\left(s_{n}^{*} Z\right)_{n \in \mathbb{N}}$ also leaves every compact subset of $S / Z$, since the involution on $S$ induces an involution ${ }^{*}: S / Z \rightarrow S / Z$. We write

$$
\rho: S \rightarrow B\left(\mathcal{B}^{2}\left(S / Z, \chi_{\lambda}\right)\right), \quad(\rho(s) . f)(z)=f(z s)
$$

for the right regular representation of $S$ on $\mathcal{B}^{2}\left(S / Z, \chi_{\lambda}\right)$ and note that $\left(\rho, \mathcal{B}^{2}\left(S / Z, \chi_{\lambda}\right)\right)$ is a holomorphic contraction representation of $S$ (cf. $\left[\right.$ Kr98, Prop. II.4]). Let $s_{0} \in S^{0}$. Then $\rho\left(s_{0}\right) \cdot K_{s_{n}}^{\lambda} \rightarrow \rho\left(s_{0}\right) \cdot f^{\lambda}$ and $\rho\left(s_{0}\right) \cdot f^{\lambda} \neq 0$ since $\rho\left(s_{0}\right)$ is an injective operator. It follows in particular that there exists a convergent subsequence of $\left(\rho\left(s_{0}\right) . K_{s_{n}}^{\lambda}\right)_{n \in \mathbb{N}}$ converging to $\rho\left(s_{0}\right) \cdot f^{\lambda}$ pointwise. Note that $\left|K_{s}^{\lambda}\right| \in C_{0}(S / Z) \mid S_{S^{0} / Z}$ for all $s \in S^{0}$ (cf. [Kr98, Prop. II.4]). Thus we obtain from

$$
\begin{aligned}
\rho\left(s_{0}\right) \cdot K_{s_{n}}^{\lambda}(z) & =K_{s_{n}}^{\lambda}\left(z s_{0}\right)=K^{\lambda}\left(z s_{0}, s_{n}\right)=K^{\lambda}\left(z, s_{n} s_{0}^{*}\right) \\
& =K^{\lambda}\left(s_{n}^{*} z, s_{0}^{*}\right)=K_{s_{0}^{*}}^{\lambda}\left(s_{n}^{*} z\right)
\end{aligned}
$$

for all $z \in S^{0}$ that $\rho\left(s_{0}\right) \cdot K_{s_{n}}^{\lambda} \rightarrow 0$ pointwise. This is a contradiction to $\rho\left(s_{0}\right) . f^{\lambda} \neq 0$ and proves our first step.

Step 2: Every cluster point of $\left(s_{n} Z\right)_{n \in \mathbb{N}}$ lies in $S^{0} / Z$.

As the Polar Decomposition of $S$ is inherited by $S / Z$, i.e., the mapping

$$
G / Z \times W \rightarrow S / Z, \quad(g Z, X) \mapsto g \operatorname{Exp}(i X) Z
$$

is a homeomorphism, we can write $s_{n}=g_{n} \operatorname{Exp}\left(X_{n}\right)$, where $g_{n} \in G$ and $X_{n} \in i W^{0}$. According to Step 1 , we now may assume that both $\left(g_{n} Z\right)_{n \in \mathbb{N}}$ and $\left(X_{n}\right)_{n \in \mathbb{N}}$ converge. Let $X \in i W$ be the limit of $\left(X_{n}\right)_{n \in \mathbb{N}}$. Note that it suffices to show that $X \in i W^{0}$. As $\left(K_{s_{n}}\right)_{n \in \mathbb{N}}$ is convergent with non-zero 
limit, $\left(\left\|K_{s_{n}}\right\|^{2}\right)_{n \in \mathbb{N}}$ is a convergent sequence in $\mathbb{R}^{+}$with positive limit. The bi-invariance of $K$ further implies that

$$
\begin{aligned}
\left\|K_{s_{n}}\right\|^{2}=K\left(s_{n}, s_{n}\right) & =K\left(g_{n} \operatorname{Exp}\left(X_{n}\right), g_{n} \operatorname{Exp}\left(X_{n}\right)\right) \\
& =K\left(\operatorname{Exp}\left(X_{n}\right), \operatorname{Exp}\left(X_{n}\right)\right),
\end{aligned}
$$

so that Step 2 follows from Theorem II.8.

(ii) This is a direct consequence of (i) and the compactness of $Z$.

Lemma III.5. If $S=\Gamma_{G}(W)$ is a pointed complex Ol'shanskiu semigroup, then there exists a finite set $F \subseteq H W\left(G, \Delta^{+}\right)$such that all representations $\pi_{K^{N}}: S \rightarrow B\left(\mathcal{H}_{K^{N}}\right), N \in \mathbb{N}$, associated to $\Gamma^{\prime}=\Gamma \cup F$ (cf. Remark II.10) are injective.

Proof. Since $S$ is pointed, it follows from [Kr98, Prop. V.7] that $\pi_{K^{N}}$ is injective if and only if $\left.\pi_{K^{N}}\right|_{T}$ is injective, where $T=\exp \mathrm{t}$. Evaluation of the operators $\pi_{K^{N}}(t), t \in T$, on highest weight vectors $v_{\lambda}, \lambda \in \Gamma\left(i\right.$ int $\left.C^{\star}, 2 \rho\right)$, now easily shows how one can choose $F$ to obtain injective representations. For more details we refer to [Ne96a, Sect. V].

Theorem III.6. Let $D \subseteq \Gamma_{G}\left(W_{\max }^{0}\right)$ be a pointed bi-invariant Stein domain and suppose that $G$ is a (CA)-Lie group and $Z$ is compact. Then there exists a kernel $K \in \mathcal{P}_{G \times G}\left(D^{2}\right)$ such that the mapping

$$
e_{K}: D \rightarrow \mathcal{H}_{K}^{*} \backslash\{0\}, \quad z \mapsto K_{z}
$$

defines a $G \times G$-equivariant holomorphic embedding with closed range.

Proof. In view of Proposition I.8(i) and Lemma I.6(ii), we may assume that $D=S^{0}=\Gamma_{G}\left(W^{0}\right)$ is a pointed open complex Ol'shanskiu semigroup. Now let $\Gamma^{\prime}$ as in Lemma III.5 and $K=K^{N}$ for some $N \in \mathbb{N}$. As $K \neq 0$, the map $e_{K}$ is a well defined $G \times G$-equivariant holomorphic map (cf. Corollary III.2). Lemma III.5 implies that the representation $\pi_{K}: S \rightarrow B\left(\mathcal{H}_{K}\right)$ is injective so that $\mathcal{H}_{K}$ separates points by [Kr98, Prop. V.10], which in turn means that $e_{K}$ is injective.

Next we show that ime $e_{K}$ is closed. In fact, if $K_{s_{n}} \rightarrow f \neq 0$, then Lemma III.4 implies that $\left(s_{n}\right)_{n \in N}$ is a bounded sequence in $S^{0}$ with all accumulation points in $S^{0}$. Thus we find a convergent subsequence $\left(s_{n_{k}}\right)_{k \in \mathbb{N}}$ with limit $s \in S^{0}$. Now we get

$$
f=\lim _{n \rightarrow \infty} K_{s_{n}}=\lim _{k \rightarrow \infty} K_{s_{n_{k}}}=\lim _{k \rightarrow \infty} e_{K}\left(s_{n_{k}}\right)=e_{K}(s)=K_{s},
$$

proving the closedness of ime $e_{K}$.

Finally another easy application of Lemma III.4 shows that $e_{K}$ is homeomorphic onto its image, concluding the proof of the theorem. 
Example III.7 (The Bergman kernel associated to $\operatorname{Sl}(2, \mathbb{R})$ ). Let $G=\operatorname{Sl}(2, \mathbb{R})$ and $\mathfrak{g}:=\mathfrak{s l}(2, \mathbb{R})$. We choose

$$
U=\left(\begin{array}{cc}
0 & 1 \\
-1 & 0
\end{array}\right), \quad T=\left(\begin{array}{ll}
0 & 1 \\
1 & 0
\end{array}\right), \quad \text { and } \quad H=\left(\begin{array}{cc}
1 & 0 \\
0 & -1
\end{array}\right)
$$

as a basis for $\mathfrak{g}$.

Then $\mathfrak{t}:=\mathbb{R} U$ is a compactly embedded Cartan subalgebra. Let $\alpha \in i \mathbf{t}^{*}$ be defined by $\alpha(U)=-2 i$. The root system of $\mathfrak{g}$ is given by $\Delta=\{ \pm \alpha\}$ with root spaces $\mathfrak{g}_{\mathbb{C}}^{\alpha}=\mathbb{C}(T+i H)$ and $\mathfrak{g}_{\mathbb{C}}^{-\alpha}=\mathbb{C}(T-i H)$. We define a positive system by $\Delta^{+}:=\{\alpha\}$ and write $\kappa$ for the Cartan-Killing form on $\mathfrak{g}$. Then the upper light cone

$$
\begin{aligned}
W: & =\{X=u U+t T+h H: u \geq 0, \kappa(X, X) \leq 0\} \\
& =\left\{X=u U+t T+h H: u \geq 0, h^{2}+t^{2}-u^{2} \leq 0\right\}
\end{aligned}
$$

is an invariant pointed cone in $\mathfrak{g}$. Moreover, $W$ is up to sign the unique invariant elliptic cone in $\mathfrak{g}$ (cf. [HiNe93, Th. 7.25]). Thus up to isomorphism $S:=\Gamma_{G}(W)$ is the unique complex Ol'shanskil semigroup corresponding to $G$.

In the following we identify $\mathfrak{t}_{\mathbb{C}}$ with $\mathbb{C}$ via the isomorphism $\mathfrak{t}_{\mathbb{C}} \rightarrow \mathbb{C}, \lambda \mapsto$ $\lambda(i U)$. Then $H W\left(G, \Delta^{+}\right)=H W(G, W)=\{\lambda \in \mathbb{Z}: \lambda \leq 0\}, \lambda+2 \rho \in i \operatorname{int} C^{\star}$ if and only if $\lambda \leq-3$, and thus $\Gamma\left(i\right.$ int $\left.C^{\star}, 2 \rho\right)=\{\lambda \in \mathbb{Z}: \lambda \leq-3\}$. The main point is that $\Gamma\left(i\right.$ int $\left.C^{\star}, 2 \rho\right)$ coincides with the weights in the decomposition of the Bergman kernel $B$ of the Bergman space

$$
\mathcal{B}^{2}(S):=\left\{f \in \operatorname{Hol}\left(S^{0}\right):\|f\|_{2}^{2}:=\int_{S^{0}}|f(s)|^{2} d \mu_{G_{\mathbb{C}}}(s)<\infty\right\}
$$

(cf. [Kr98, Th. IV.7]). Further one knows that

$$
B=\sum_{\lambda \leq-3} \lambda(1+\lambda)^{2}\left(4-\lambda^{2}\right) K^{\lambda}
$$

(cf. [Kr98, Th. IV.7, Ex. IV.8]) so that Theorem II.8 and Theorem III.6 imply that

and that the map

$$
\lim _{z \rightarrow \partial S} B(z, z)=\infty
$$

$$
e_{B}: S^{0} \rightarrow \mathcal{B}^{2}(S)^{*} \backslash\{0\}, \quad s \mapsto B_{s}
$$

is a $G \times G$-equivariant holomorphic embedding with closed range.

Problems III.8. (a) What is the reason for that one has to exclude zero in $\mathcal{H}_{K}^{*}$ to obtain the closedness of the map $e_{K}$ ?

(b) Given a pointed biinvariant Stein domain $D$, does there exist an equivariant closed embedding of $D$ into a complex topological vector space $E$ endowed with a continuous $G \times G$-action? 


\section{References}

[GG77] M. Gel'fand and S.G. Gindikin, Complex manifolds whose skeletons are real Lie groups, and analytic discrete series of representations, Funct. Anal. and Appl., 11 (1977), 19-27.

[HHL89] J. Hilgert, K.H. Hofmann and J.D. Lawson, Lie Groups, Convex Cones, and Semigroups, Oxford University Press, 1989.

[HiNe93] J. Hilgert and K.-H. Neeb, Lie semigroups and their Applications, Lecture Notes in Math., 1552, Springer, 1993.

[HiNe96] _ Positive definite spherical functions on Ol'shanskiu domains, Mathematik-Bericht, 96/7, Technische Universität Clausthal.

[Hö73] L. Hörmander, An introduction to complex analysis in several variables, NorthHolland, 1973.

[Kr97] B. Krötz, The Plancherel theorem for bi-invariant Hilbert spaces, submitted.

[Kr98] _ On Hardy and Bergman spaces on complex Ol'shanskiu semigroups, Math. Ann., 312 (1998), 13-52.

[KrNe96] B. Krötz and K.-H. Neeb, On hyperbolic cones and mixed symmetric spaces, Journal of Lie Theory, 6(1) (1996), 69-146.

[Ne94] K.-H. Neeb, Holomorphic representation theory II, Acta Math., 173(1) (1994), 103-133.

[Ne96a] _ Coherent states, holomorphic extensions, and highest weight representations, Pac. J. Math., 174(2) (1996), 497-542.

[Ne96b] - Invariant convex sets and functions in Lie algebras, Semigroup Forum, 53 (1996), 230-261.

[Ne97]_ Representation Theory and Convexity, submitted.

[Ne98] _ On the complex and convex geometry of Ol'shanskiu semigroups, Ann. Inst. Fourier, 48(1) (1998), 149-203.

[Ne99] Holomorphy and Convexity in Lie Theory, Expositions in Mathematics, de Gruyter, in preparation.

Received September 22, 1997 and revised November 20, 1997.

TU Clausthal

ERZSTRASSE 1

D-38678 Clausthal-ZELlerfeld

GERMANY

E-mail address: mabk@math.tu-clausthal.de 